\title{
Monochromatic and zero-sum sets of nondecreasing modified diameter
}

\author{
David Grynkiewicz*and Rasheed Sabar ${ }^{\dagger}$ \\ Submitted: Oct 24, 2004; Accepted: Mar 24, 2006; Published: Mar 30, 2006 \\ Mathematics Subject Classification: 05D05 (11B75)
}

\begin{abstract}
Let $m$ be a positive integer whose smallest prime divisor is denoted by $p$, and let $\mathbb{Z}_{m}$ denote the cyclic group of residues modulo $m$. For a set $B=\left\{x_{1}, x_{2}, \ldots, x_{m}\right\}$ of $m$ integers satisfying $x_{1}<x_{2}<\cdots<x_{m}$, and an integer $j$ satisfying $2 \leq j \leq m$, define $g_{j}(B)=x_{j}-x_{1}$. Furthermore, define $f_{j}(m, 2)$ (define $f_{j}\left(m, \mathbb{Z}_{m}\right)$ ) to be the least integer $N$ such that for every coloring $\Delta:\{1, \ldots, N\} \rightarrow\{0,1\}$ (every coloring $\left.\Delta:\{1, \ldots, N\} \rightarrow \mathbb{Z}_{m}\right)$, there exist two $m$-sets $B_{1}, B_{2} \subset\{1, \ldots, N\}$ satisfying: (i) $\max \left(B_{1}\right)<\min \left(B_{2}\right)$, (ii) $g_{j}\left(B_{1}\right) \leq g_{j}\left(B_{2}\right)$, and (iii) $\left|\Delta\left(B_{i}\right)\right|=1$ for $i=1,2$ (and (iii) $\sum_{x \in B_{i}} \Delta(x)=0$ for $\left.i=1,2\right)$. We prove that $f_{j}(m, 2) \leq 5 m-3$ for all $j$, with equality holding for $j=m$, and that $f_{j}\left(m, \mathbb{Z}_{m}\right) \leq 8 m+\frac{m}{p}-6$. Moreover, we show that $f_{j}(m, 2) \geq 4 m-2+(j-1) k$, where $k=\left\lfloor\left(-1+\sqrt{\frac{8 m-9+j}{j-1}}\right) / 2\right\rfloor$, and, if $m$ is prime or $j \geq \frac{m}{p}+p-1$, that $f_{j}\left(m, \mathbb{Z}_{m}\right) \leq 6 m-4$. We conclude by showing $f_{m-1}(m, 2)=f_{m-1}\left(m, \mathbb{Z}_{m}\right)$ for $m \geq 9$.
\end{abstract}

\section{Introduction}

Let $[a, b]$ denote the set of integers between $a$ and $b$, inclusive. For a set $S$, an $S$-coloring of $[1, N]$ is a function $\Delta:[1, N] \rightarrow S$. If $S=\{0,1, \ldots, r-1\}$, then we call $\Delta$ an $r$-coloring. The following is the Erdös-Ginzburg-Ziv (EGZ) theorem, [1] [14] [30].

Theorem 0. Let $m$ be a positive integer. If $\Delta:[1,2 m-1] \rightarrow \mathbb{Z}_{m}$, then there exist distinct integers $x_{1}, x_{2}, \ldots, x_{m} \in[1,2 m-1]$ such that $\sum_{i=1}^{m} \Delta\left(x_{i}\right)=0$. Moreover, $2 m-1$ is the smallest number for which the above assertion holds.

\footnotetext{
*Department of Mathematics, Caltech, Pasadena, CA, 91125

${ }^{\dagger}$ Department of Mathematics, Harvard University, Cambridge, MA, 02138

‡The second author was funded by NSF grant DMS0097317.
} 
The EGZ theorem can be viewed as a generalization of the pigeonhole principle for 2 boxes (since the $m$-term zero-sum subsequences of a sequence consisting only of 0 's and 1's are exactly the monochromatic $m$-term subsequences). As such, several theorems of Ramsey-type have been generalized similarly by considering $\mathbb{Z}_{m}$-colorings and zerosum configurations rather than 2-colorings and monochromatic configurations. When in such a theorem the size of the configuration needed to guarantee a monochromatic sub-configuration equals the size of the configuration needed to guarantee a zero-sum sub-configuration (as it does for the pigeonhole principle versus EGZ), we say that the theorem zero-sum generalizations. The most well known such theorem is the zero-trees theorem [17] [33]. Two surveys of related results and open problems appear in [3] [12], and some examples of other various extensions of EGZ appear in [10] [11] [16] [18] [19] [20] [21] [27] [31] [32].

One of the first Ramsey-type problems considered with respect to zero-sum generalizations was the nondecreasing diameter problem introduced by Bialostocki, Erdős, and Lefmann [8]. For a set $B=\left\{x_{1}, x_{2}, \ldots, x_{m}\right\}$ of $m$ positive integers satisfying $x_{1}<x_{2}<\cdots<x_{m}$, and an integer $j$ satisfying $2 \leq j \leq m$, let $g_{j}(B)=x_{j}-x_{1}$. Note that when $j=m$, then $g_{m}(B)$ is the diameter of the set $B$. Let $f_{j}(m, 2)$ (let $f_{j}\left(m, \mathbb{Z}_{m}\right)$ ) be the least integer $N$ such that for every coloring $\Delta:[1, N] \rightarrow\{0,1\}$ (for every coloring $\Delta:[1, N] \rightarrow \mathbb{Z}_{m}$ ), there exist two $m$-sets $B_{1}, B_{2} \subset[1, N]$ satisfying (i) $\max \left(B_{1}\right)<\min \left(B_{2}\right)$, (ii) $g_{j}\left(B_{1}\right) \leq g_{j}\left(B_{2}\right)$, and (iii) $\left|\Delta\left(B_{i}\right)\right|=1$ for $i=1,2$ (and (iii) $\sum_{x \in B_{i}} \Delta(x)=0$ for $\left.i=1,2\right)$. Bialostocki, Erdős, and Lefmann introduced the functions $f_{m}(m, 2)$ and $f_{m}\left(m, \mathbb{Z}_{m}\right)$ and showed that $f_{m}(m, 2)=f_{m}\left(m, \mathbb{Z}_{m}\right)=5 m-3$, thus obtaining one of the first 2-color zero-sum generalizations for a Ramsey-type problem [8]. They also introduced a notion of zero-sum generalization for Ramsey-type problems involving arbitrary $r$-colorings (not just 2-colorings), and showed that the corresponding 3-color version of the nondecreasing diameter problem for two $m$-sets also zero-sum generalized. Recently, the four color case was shown to zero-sum generalize [24], but the cases with $r>4$ remain open and difficult.

In this paper we introduce and study the functions $f_{j}(m, 2)$ and $f_{j}\left(m, \mathbb{Z}_{m}\right)$ with $j<m$, thus studying the nondecreasing diameter problem by varying the notion of diameter by the parameter $j$. One of our main tools is an improvement to a recent generalization (Theorem 2.7) of results of Mann [29], Olson [31], Bollobás and Leader [10], and Hamidoune [26], that was developed by the first author [23] while studying the original nondecreasing diameter problem for four colors [24].

For a positive integer $m$, let $F(m, 2)=\max \left\{f_{j}(m, 2) \mid 2 \leq j \leq m\right\}$ and let $F\left(m, \mathbb{Z}_{m}\right)=$ $\max \left\{f_{j}\left(m, \mathbb{Z}_{m}\right) \mid 2 \leq j \leq m\right\}$. This project was begun when A. Bialostocki suggested the following two conjectures [2].

\section{Conjecture 1.1.}

$$
\liminf _{m \rightarrow \infty} \frac{F\left(m, \mathbb{Z}_{m}\right)}{F(m, 2)}=1
$$


Conjecture 1.2. If $j \geq 2$ is an integer, then

$$
\liminf _{m \rightarrow \infty} \frac{f_{j}\left(m, \mathbb{Z}_{m}\right)}{f_{j}(m, 2)}=1
$$

and

$$
\liminf _{m \rightarrow \infty} \frac{f_{m-j}\left(m, \mathbb{Z}_{m}\right)}{f_{m-j}(m, 2)}=1,
$$

Among other results, we support Conjecture 1.1, proving that $\liminf _{m \rightarrow \infty} \frac{F\left(m, \mathbb{Z}_{m}\right)}{F(m, 2)} \leq 1.2$. Furthermore, we prove the case $j=1$ for the second part of Conjecture 1.2 by showing that

$$
f_{m-1}(m, 2)=f_{m-1}\left(m, \mathbb{Z}_{m}\right)=5 m-4 \quad \text { for } m \geq 9 .
$$

The paper is organized as follows. Section 2 contains definitions, terminology, and results used in Sections 3 and 4, which contain results addressing Conjectures 1.1 and 1.2, respectively.

\section{Preliminaries}

We recall some theorems from additive number theory, but first we need to introduce terminology used in [23] and [30]. If $G$ is an abelian group and $A, B \subseteq G$, then their sumset is $A+B=\{a+b \mid a \in A, b \in B\}$. A set $A \subseteq G$ is said to be $H$-periodic, if it is the union of $H$-cosets for some nontrivial subgroup $H$ of $G$, and otherwise, $A$ is called aperiodic. We say that $A$ is maximally $H$-periodic, if $A$ is $H$-periodic, and $H$ is the maximal subgroup for which $A$ is periodic; in this case, $H=\{x \in G \mid x+A=A\}$, and $H$ is sometimes referred to as the stabilizer of $A$. If $S$ is a sequence of elements from $G$, then an $n$-set partition of $S$ is a partition of the sequence $S$ into $n$ nonempty subsequences, $A_{1}, \ldots, A_{n}$, such that the terms in each subsequence $A_{i}$ are all distinct (thus allowing each subsequence $A_{i}$ to be considered a set). A sequence of elements from $\mathbb{Z}_{m}$ is zero-sum if the sum of its terms is zero. An affine transformation is any map $\gamma: \mathbb{Z}_{m} \rightarrow \mathbb{Z}_{m}$ given by $\gamma(x)=k x+b$, where $k, b \in \mathbb{Z}_{m}$ and $\operatorname{gcd}(k, m)=1$. Furthermore, $|S|$ denotes the cardinality of $S$, if $S$ is a set, and the length of $S$, if $S$ is a sequence. If $S$ is an ordered set and $r$ is an integer satisfying $|S| \geq r$, then elements $y_{1}<y_{2}<\cdots<y_{r} \in S$ are said to be a final segment if $y_{i}=\max \left(S \backslash\left\{y_{i+1}, y_{i+2}, \ldots, y_{r}\right\}\right)$ for $i=1,2, \ldots, r$. Analogously, integers $y_{1}<y_{2}<\cdots<y_{r} \in S$ are said to be an initial segment if $y_{i}=\min \left(S \backslash\left\{y_{i-1}, y_{i-2}, \ldots, y_{1}\right\}\right)$ for $i=1,2, \ldots, r$. Finally, for $j \in \mathbb{Z}_{m}$, we denote by $\bar{j}$ the least non-negative integer representative of $j$.

Next, we introduce helpful notation and terminology dealing specifically with our problem. Let $S_{1}$ and $S_{2}$ be sequences. Then $S_{1} \cup S_{2}$ denotes the concatenation of $S_{1}$ with $S_{2}$, and if $S_{2}$ is a subsequence of $S_{1}$, then $S_{1} \backslash S_{2}$ denotes the sequence obtained from $S_{1}$ by deleting the terms from $S_{2}$. Let $\Delta: S \rightarrow C$ be a $C$-coloring of the set $S$. If $S^{\prime} \subseteq S$, 
then we will regard $\Delta\left(S^{\prime}\right)$ as a set, and if $x \in S$, then we regard $\Delta(x)$ as an element. The sequence of colors given by $\Delta$ will often be abbreviated as a string using exponential notation (e.g. the sequence given by the coloring $\Delta([1,3])=\{1\}, \Delta([4,7])=\{2\}$ is abbreviated by $\left.1^{3} 2^{4}\right)$. We use $\Delta S$ to denote the sequence of colors for $S$ given by $\Delta$ (hence $\Delta[1,7]=1^{3} 2^{4}$ in the previous example). If $c \in C$ and $\Delta^{-1}(c)=\left\{x_{1}, x_{2}, \ldots, x_{s}\right\}$, where $x_{1}<x_{2}<\cdots<x_{s}$, then for an integer $r \leq s$, define

$$
\Pi(r, c)=x_{r} \quad \text { and } \quad \amalg(r, c)=x_{s-r+1} .
$$

Let $\Delta: S \rightarrow \mathbb{Z}_{m}$ be a coloring of the set $S$. A set $B \subset S$ is zero-sum if $\sum_{x \in B} \Delta(x)=0$. Further, $\Delta$ is said to reduce to monochromatic if either $|\Delta(S)| \leq 2$ or there exists $B \subset S$ such that $|B| \leq m-1$ and $|\Delta(S \backslash B)|=1$. Observe that in either case there exists a natural induced coloring $\Delta^{*}: S \rightarrow\{0,1\}$ such that every $m$-element monochromatic set under $\Delta^{*}$ is zero-sum under $\Delta$. Finally, let $m$ and $j$ be integers satisfying $2 \leq j \leq m$, and let $\Delta: S \rightarrow\{0,1\}$ (let $\Delta: S \rightarrow \mathbb{Z}_{m}$ ) be a coloring. Then two $m$-sets $B_{1}, B_{2} \subset S$ are said to be an $(m, j)$-solution (an $\left(m, j, \mathbb{Z}_{m}\right)$-solution) if $\max \left(B_{1}\right)<\min \left(B_{2}\right), g_{j}\left(B_{1}\right) \leq g_{j}\left(B_{2}\right)$, and $\left|\Delta\left(B_{1}\right)\right|=\left|\Delta\left(B_{2}\right)\right|=1$ (and $\sum_{x \in B_{i}} \Delta(x)=0$ for $i=1,2$ ).

First we state a theorem, which is an easy consequence of the Pigeonhole Principle, sometimes referred to as the Caveman Theorem since its roots extend back so far [15].

Theorem 2.1. Let $S$ be a sequence of elements from a finite abelian group $G$. If $|S|=|G|$, then there exists a nonempty zero-sum subsequence consisting of consecutive terms of $S$.

The following theorem is the Cauchy-Davenport Theorem [30] [13].

Theorem 2.2. Let $m$ be a prime and let $n$ be a positive integer. If $A_{1}, A_{2}, \ldots, A_{n}$ is a collection of subsets of $\mathbb{Z}_{m}$, then

$$
\left|\sum_{i=1}^{n} A_{i}\right| \geq \min \left\{m, \sum_{i=1}^{n}\left|A_{i}\right|-n+1\right\} .
$$

Next, we will need the following slightly stronger form of the EGZ theorem [12].

Theorem 2.3. Let $k, m$ be positive integers such that $k \mid m$. If $\Delta:[1, m+k-1] \rightarrow \mathbb{Z}_{m}$, then there exist distinct integers $x_{1}, x_{2}, \ldots, x_{m} \in[1, m+k-1]$ such that $\sum_{i=1}^{m} \Delta\left(x_{i}\right) \equiv 0$ $\bmod k$. Moreover, $m+k-1$ is the smallest number for which the above assertion holds.

The following theorem turns out to be useful. The proofs of parts (a) and (b) appear in [5] and [9] [7], respectively.

Theorem 2.4. Let $m \geq 4$ be an integer, and let $\Delta: S \rightarrow \mathbb{Z}_{m}$ be a coloring of a set of integers $S$ for which $|\Delta(S)| \geq 3$.

(a) If $|S|=2 m-2$, then there exist distinct integers $x_{1}, \ldots, x_{m}$ such that $\sum_{i=1}^{m} \Delta\left(x_{i}\right)=0$. (b) If $|S|=2 m-3$, and there are not distinct integers $x_{1}, \ldots, x_{m}$ such that $\sum_{i=1}^{m} \Delta\left(x_{i}\right)=$ 0 , then $\Delta(S)=\{a, b, c\}$, where $\left|\Delta^{-1}(a)\right|=m-1,\left|\Delta^{-1}(b)\right|=m-3$, and $\left|\Delta^{-1}(c)\right|=1$; moreover, up to affine transformation we may assume that $a=0, b=1$, and $c=2$. 
The following simple proposition will be helpful [7].

Proposition 2.5. Let $S$ be a sequence of elements from a finite abelian group $G$, and let $A=A_{1}, \ldots, A_{n}$ be an $n$-set partition of $S$, where $\left|\sum_{i=1}^{n} A_{i}\right|=r>1$. Then there exists a subsequence $S^{\prime}$ of $S$ and an $n^{\prime}$-set partition $A^{\prime}=A_{j_{1}}, \ldots, A_{j_{n^{\prime}}}$ of $S^{\prime}$, which is a subsequence of the $n$-set partition $A=A_{1}, \ldots, A_{n}$, such that $n^{\prime} \leq r-1$ and $\left|\sum_{i=1}^{n^{\prime}} A_{j_{i}}\right|=r$.

Before stating the next two theorems, we provide a few remarks to clarify an otherwise nebulous and complicated time-line. The main result from [23] along with its corollary first appeared, in a slightly weaker form, in the first author's undergraduate thesis. Subsequently, Theorem 2.7 was obtained for this collaborative article as a means of augmenting the weaker version of the corollary in [23]. Later, the strengthening for both results from [23] was found by the first author and incorporated into the final version of [23]. However, the new proofs for the result from [23] almost immediately gave a generalization of Theorem 2.7, as noted in [22]. Unfortunately, due to the idiosyncracies of the publishing world, the results in [23] and [22], despite being historically newer, were both published before this article, which predate them. Consequently, the original (and much more complicated) proof of Theorem 2.7 now seems unnecessary, and has been omitted. Instead we derive Theorem 2.7 from Theorem 2.6 [22].

Theorem 2.6. Let $S^{\prime}$ be a subsequence of a finite sequence $S$ of terms from an abelian group $G$ of order $m$, let $P=P_{1}, \ldots, P_{n}$ be an n-set partition of $S^{\prime}$, let $a_{i} \in P_{i}$ for $i \in\{1, \ldots, n\}$, and let $p$ be the smallest prime divisor of $m$. If $n \geq \min \left\{\frac{m}{p}-1, \frac{\left|S^{\prime}\right|-n+1}{p}-1\right\}$, then either:

(i) there is an $n$-set partition $A=A_{1}, \ldots, A_{n}$ of a subsequence $S^{\prime \prime}$ of $S$ with $\left|S^{\prime}\right|=\left|S^{\prime \prime}\right|$, $\sum_{i=1}^{n} P_{i} \subseteq \sum_{i=1}^{n} A_{i}, a_{i} \in A_{i}$ for $i \in\{1, \ldots, n\}$, and

$$
\left|\sum_{i=1}^{n} A_{i}\right| \geq \min \left\{m,\left|S^{\prime}\right|-n+1\right\},
$$

(ii) there is a proper, nontrivial subgroup $H_{a}$ of index $a$, a coset $\alpha+H_{a}$ such that all but e terms of $S$ are from $\alpha+H_{a}$, where

$$
e \leq \min \left\{a-2,\left\lfloor\frac{\left|S^{\prime}\right|-n}{\left|H_{a}\right|}\right\rfloor-1\right\}
$$

an $n$-set partition $A=A_{1}, \ldots, A_{n}$ of of subsequence $S^{\prime \prime}$ of $S$ with $\left|S^{\prime \prime}\right|=\left|S^{\prime}\right|, \sum_{i=1}^{n} P_{i} \subseteq \sum_{i=1}^{n} A_{i}$, $a_{i} \in A_{i}$ for $i \in\{1, \ldots, n\}$, and $\left|\sum_{i=1}^{n} A_{i}\right| \geq(e+1)\left|H_{a}\right|$, and an $n$-set partition $B=B_{1}, \ldots, B_{n}$ of a subsequence $S_{0}^{\prime \prime}$ of $S$, with all terms of $S_{0}^{\prime \prime}$ from $\alpha+H_{a}$ and $\left|S_{0}^{\prime \prime}\right| \leq n+\left|H_{a}\right|-1$, such that $\sum_{i=1}^{n} B_{i}=n \alpha+H_{a}$. 
Theorem 2.7. Let $S$ be a sequence of elements from an abelian group $G$ of order $m$ with an $n$-set partition $P=P_{1}, \ldots, P_{n}$, and let $p$ be the smallest prime divisor of $m$. Suppose that $n^{\prime} \geq \frac{m}{p}-1$, that $|S| \geq m+\frac{m}{p}+p-3$, and that $P$ has at least $n-n^{\prime}$ cardinality one sets. Then either:

(i) there exists an $n$-set partition $A=A_{1}, A_{2}, \ldots, A_{n}$ of $S$ with at least $n-n^{\prime}$ cardinality one sets, such that:

$$
\left|\sum_{i=1}^{n} A_{i}\right| \geq \min \{m,|S|-n+1\}
$$

(ii) (a) there exists $\alpha \in G$ and a nontrivial proper subgroup $H_{a}$ of index a such that all but at most $\min \left\{a-2,\left|\frac{|S|-n}{\left|H_{a}\right|}\right|-1\right\}$ terms of $S$ are from the coset $\alpha+H_{a}$; and $(b)$ there exists an $n$-set partition $A_{1}, A_{2}, \ldots, A_{n}$ of the subsequence of $S$ consisting of terms from $\alpha+H_{a}$ such that $\sum_{i=1}^{n} A_{i}=n \alpha+H_{a}$.

Proof. Let $S^{\prime}$ be the sequence partitioned by the $n^{\prime}$-set partition $P_{1}, \ldots, P_{n^{\prime}}$. Apply Theorem 2.6 to $S^{\prime}$ with $n^{\prime}=n$. If Theorem 2.6(i) holds, then (i) follows by appending the remaining $n-n^{\prime}$ elements of $S$ as singleton sets. Otherwise, Theorem 2.6(ii) implies (ii) by replacing the elements of $S$ removed from the $B_{i}$ and appending on $n-n^{\prime}$ elements from the coset $\alpha+H_{a}$ as singleton sets (possible in view of the existence of the set partition $A$, in fact, the proof of Theorem 2.6 obtains the set partition $B$ by removing elements from a set partition satisfying Theorem 2.7(ii)).

\section{General upper and lower bounds}

Theorem 3.1. Let $m, j$ be integers with $2 \leq j \leq m$, and let $k=\left\lfloor\left(-1+\sqrt{\frac{8 m-9+j}{j-1}}\right) / 2\right\rfloor$. Then $f_{j}(m, 2) \geq 4 m-2+(j-1) k$.

Proof. Consider the coloring $\Delta:[1,4 m-3+(j-1) k] \rightarrow\{0,1\}$ given by

$$
0^{m-1-(j-1) \frac{k(k+1)}{2}}\left(1^{j-1} 0^{k(j-1)}\right)\left(1^{j-1} 0^{(k-1)(j-1)}\right) \cdots\left(1^{j-1} 0^{2(j-1)}\right)\left(1^{j-1} 0^{j-1}\right) 1^{2 m-1} 0^{m-1} .
$$

Using the quadratic formula, it can be easily verified that $k$ is the greatest integer such that $\sum_{i=1}^{k}(j-1) i=(j-1) \frac{k(k+1)}{2} \leq m-1$. Thus,

$$
\left|\Delta^{-1}(0) \cap[1, m-1+(j-1) k]\right|=m-1,
$$

and

$$
\left|\Delta^{-1}(1) \cap[1, m-1+(j-1) k]\right|=(j-1) k \leq m-1 .
$$

Suppose there exist sets $B_{1}, B_{2}$ which are an $(m, j)$-solution. Notice that $\Delta\left(B_{1}\right) \neq\{0\}$, since otherwise $\left|\left[\max \left(B_{1}\right)+1,4 m-3+(j-1) k\right]\right| \leq m-2$. Similarly, $\Delta\left(B_{2}\right) \neq\{0\}$. Thus $\Delta\left(B_{i}\right)=\{1\}$ for $i=1,2$. Furthermore, given any $m$-set $B$ with $\Delta(B)=\{1\}$, there 
exists an $m$-set $B^{*}$ with $\Delta\left(B^{*}\right)=\{1\}$ satisfying $\max \left(B^{*}\right) \leq \max (B), g_{j}\left(B^{*}\right) \leq g_{j}(B)$, and $(j-1) \mid g_{j}\left(B^{*}\right)$ (simply compress the set $B$ inwards until the first $j$ integers are consecutive with the exception of one gap of length $t(j-1)$ where a single block of zero's prevents further compression). Therefore we may assume $g_{j}\left(B_{1}\right)=j-1+t(j-1)$ for some $t \in\{0,1, \ldots, k\}$. Since $\max \left(B_{1}\right)<\min \left(B_{2}\right)$, it follows that $B_{2}$ is contained within the last $2 m-1+t(j-1)-m$ integers colored by 1 , i.e. that

$$
B_{2} \subset \Delta^{-1}(1) \cap[\amalg(m-1+(j-1) t, 1), 4 m-3+(j-1) k] .
$$

Hence, since $\left|\Delta^{-1}(1) \cap[1, m-1+(j-1) k]\right|=(j-1) k \leq m-1$ forces $B_{2}$ to be contained in the block of $2 m-1$ consecutive integers colored by 1 , it follows that

$$
g_{j}\left(B_{2}\right) \leq(j-1)+(m-1+(j-1) t)-m=(t+1)(j-1)-1 .
$$

Consequently, $g_{j}\left(B_{1}\right)>g_{j}\left(B_{2}\right)$, a contradiction.

Remark: Theorem 3.1 yields the lower bounds $f_{m}(m, 2) \geq 5 m-3$ and $f_{m-1}(m, 2) \geq$ $5 m-4$. It is shown in [8] that the former lower bound is sharp, and we show in this paper that the latter lower bound is sharp for $m \geq 9$ as well. Therefore, the construction given in Theorem 3.1 is the best possible in some (though not all) cases.

Lemma 3.2. Let $m, j$ be integers satisfying $2 \leq j \leq m$. If $\Delta:[1,3 m-2] \rightarrow\{0,1\}$ is an arbitrary coloring, then one of the following holds:

(i) there exists a monochromatic $m$-set $B \subset[1,3 m-2]$ satisfying $g_{j}(B) \geq m+j-2$,

(ii) there exists an $(m, j)$-solution,

(iii) the coloring $\Delta$ is given (up to symmetry) by $1^{r} 0 H$, for some $r \in[j, m-1]$, and there exists a monochromatic $m$-set $B \subset 0 H$ for which $g_{j}(B) \geq m+2 j-r-3$.

Proof. Assume w.l.o.g. $\Delta(1)=1$. If $\left|\Delta^{-1}(1)\right|<m$, then $\left|\Delta^{-1}(0)\right| \geq 2 m-1$, whence (i) follows. So $\left|\Delta^{-1}(1)\right| \geq m$. Let $S=[m+j-1,3 m-2]$. Since $\Delta(1)=1$ and $\left|\Delta^{-1}(1)\right| \geq m$, it follows that if $\left|\Delta^{-1}(1) \cap S\right| \geq m-j+1$, then (i) follows. Hence $\left|\Delta^{-1}(1) \cap S\right| \leq m-j$, whence

$$
\left|\Delta^{-1}(0) \cap S\right| \geq m \text {. }
$$

Let $y_{2}<y_{3}<\cdots<y_{m} \in \Delta^{-1}(0) \cap S$ be a final segment. Observe, since $\left|\Delta^{-1}(1) \cap S\right| \leq$ $m-j$, that $y_{j} \geq m+2 j-2$. Hence, if there exists $i \in[1, j]$ such that $\Delta(i)=0$, then (i) follows. Consequently, $\Delta(i)=1$ for $i \in[1, j]$. However, if $\Delta(i)=1$ for $i \in[1, m]$, then (ii) follows in view of (1). Therefore, there exists a minimal $i \in[j+1, m]$ such that $\Delta(i)=0$. Define $r=i-1$. Then the set $B=\left\{r+1, y_{2}, \ldots, y_{m}\right\}$ satisfies $g_{j}(B) \geq$ $m+2 j-2-(r+1)=m+2 j-r-3$, whence (iii) follows.

Theorem 3.3. Let $m, j$ be integers satisfying $2 \leq j \leq m$. Then $f_{j}(m, 2) \leq 5 m-3$. 
Proof. Let $\Delta:[1,5 m-3] \rightarrow\{0,1\}$ be an arbitrary coloring. Apply Lemma 3.2 to the interval $[2 m, 5 m-3]$. If Lemma 3.2(ii) holds, then the proof is complete, and if Lemma 3.2 (i) holds, then by applying the pigeonhole principle to $[1,2 m-1]$ the proof is also complete. Thus we may assume Lemma 3.2(iii) holds, so that w.l.o.g.

$$
\Delta[2 m, 5 m-3]=1^{r} 0 H,
$$

where $r$ and $H$ are as in Lemma 3.2(iii), and that there is a monochromatic subset $B \subset[2 m+r, 5 m-3]$ with $g_{j}(B) \geq m+2 j-r-3$. Let $S=[1,2 j-1]$.

Case 1: $\left|\Delta^{-1}(1) \cap S\right| \geq j$.

Since $r \leq m-1$, it follows that $g_{j}(B) \geq 2 j-2$. Hence we may assume

$$
\left|\Delta^{-1}(1) \cap[1,2 m+r-1]\right| \leq m-1 .
$$

But then since $\Delta([2 m, 2 m+r-1])=\{1\}$, it follows that

$$
\left|\Delta^{-1}(1) \cap[2 j, 2 m-1]\right| \leq m-j-r-1,
$$

implying, since $j \leq r$, that

$$
\left|\Delta^{-1}(0) \cap[2 j, 2 m-1]\right| \geq m-j+r+1 \geq m .
$$

Let $y_{1}, y_{2}, \ldots, y_{m} \in \Delta^{-1}(0) \cap[2 j, 2 m-1]$ be an initial segment. Then by (2), it follows that $B_{1}=\left\{y_{1}, \ldots, y_{m}\right\}$ is a monochromatic $m$-set with $g_{j}\left(B_{1}\right) \leq m-r-2$, whence $B_{1}, B$ are an $(m, j)$-solution.

Case 2: $\left|\Delta^{-1}(0) \cap S\right| \geq j$.

It follows, as in Case 1, that

$$
\left|\Delta^{-1}(0) \cap[1,2 m+r-1]\right| \leq m-1 .
$$

Let $d$ be the positive integer such that $r$ is contained in the interval

$$
m+j-1-\frac{m-1}{d} \leq r<m+j-1-\frac{m-1}{d+1} ;
$$

note, since

$$
\lim _{d \rightarrow \infty}\left(m+j-1-\frac{m-1}{d}\right)=m+j-1>m,
$$

and since in view of Lemma 3.2(iii) we have $j \leq r<m$, it follows that such a $d$ exists. Also note that if $j \geq \frac{m}{d}$, then (4) implies $m-1<r$, a contradiction. Hence we may assume $j<\frac{m}{d}$. From (3) and (4), it follows that

$$
\left|\Delta^{-1}(1) \cap[1,2 m+r-1]\right| \geq m+r \geq m+\left(m+j-1-\frac{m-1}{d}\right) .
$$


But then, letting $b$ be the $m-j+1$ greatest integer colored by 1 in $[1,2 m+r-1]$, since $j<\frac{m}{d}$, it follows from (5) that

$$
\left|\Delta^{-1}(1) \cap[1, b]\right| \geq m+j-1-\frac{m}{d}+j=(d-1) \frac{m}{d}+2(j-1)+1 \geq(d+1)(j-1)+1 .
$$

Hence let $z_{1}<z_{2}<\cdots<z_{m-j} \in\left\{\Delta^{-1}(1) \cap[1,2 m+r-1]\right\}$ be a final segment, and let $y_{1}<y_{2}<\cdots<y_{(d+1)(j-1)+1} \in\left\{\Delta^{-1}(1) \cap[1,2 m+r-1]\right\}$ be an initial segment. If for some index $i \in[0, d]$

$$
\left|\Delta^{-1}(0) \cap\left[y_{i(j-1)+1}, y_{(i+1)(j-1)+1}\right]\right| \leq m+j-r-2,
$$

then $B_{1}=\left\{y_{i(j-1)+1}, y_{i(j-1)+2}, \ldots, y_{(i+1)(j-1)+1}, z_{1}, z_{2}, \ldots, z_{m-j}\right\}$ is a monochromatic $m$-set with $g_{j}\left(B_{1}\right) \leq m+2 j-r-3=g_{j}(B)$, whence $B_{1}, B$ are an $(m, j)$-solution, and the proof is complete. Therefore, we may assume that

$$
\left|\Delta^{-1}(0) \cap\left[y_{i(j-1)+1}, y_{(i+1)(j-1)+1}\right]\right| \geq m+j-r-1 \quad \text { for } i=0,1, \ldots, d .
$$

But then the above inequalities and (4) imply that

$$
\left|\Delta^{-1}(0) \cap[1,2 m+r-1]\right| \geq(d+1)(m+j-r-1)>m-1,
$$

contradicting (3), and completing the proof.

Corollary 3.4. $F(m, 2)=5 m-3$.

Proof. Theorem 3.1 with $j=m$ implies that $f_{m}(m, 2) \geq 5 m-3$, whence $F(m, 2) \geq 5 m-3$. Theorem 3.3 implies that $F(m, 2) \leq 5 m-3$, as needed.

Lemma 3.5. Let $m, j$ be integers satisfying $2 \leq j \leq m$, and let $\Delta:[1,4 m-3] \rightarrow \mathbb{Z}_{m}$ be an arbitrary coloring.

(i) If $m$ is prime, then there exists a zero-sum m-set $B \subset[1,4 m-3]$ with $g_{j}(B) \geq m+j-2$;

(ii) If $j \geq \frac{m}{p}+p-1$, where $p$ is the smallest prime divisor of $m$, then there exists a zero-sum $m$-set $B \subset[1,4 m-3]$ with $g_{j}(B) \geq m+j-2$.

Proof. Consider the interval $S=[m+1,4 m-3]$. If there does not exist a $(2 m-2)$-set partition of the sequence $\Delta S$ with $m-1$ sets of cardinality 2 , then since $|S|=3 m-3$, it follows that there exists $a \in \mathbb{Z}_{m}$ such that

$$
\left|\Delta^{-1}(a) \cap S\right| \geq 2 m-1 \quad \text { and } \quad\left|\Delta^{-1}\left(\mathbb{Z}_{m} \backslash\{a\}\right) \cap S\right| \leq m-2
$$

Let $y_{1}<y_{2}<\cdots<y_{2 m-1} \in \Delta^{-1}(a) \cap S$ and $B=\left\{y_{1}, \ldots, y_{j-1}, y_{m+j-1}, y_{m+j}, \ldots, y_{2 m-1}\right\}$. Then $g_{j}(B) \geq m+j-2$, and the proof is complete. So we may assume that there exists a $(2 m-2)$-set partition $P$ of the sequence $\Delta S$ with $(m-1)$ sets of cardinality 2 .

Suppose first that $m$ is prime. Define $x_{1}=1$. Applying the Cauchy-Davenport theorem to $P$, it follows that there exist integers $x_{2}<x_{3}<\cdots<x_{m} \in S$ such that 
$\sum_{i=2}^{m} \Delta\left(x_{i}\right)=-\Delta\left(x_{1}\right)$. Thus, $\left(x_{1}, \ldots, x_{m}\right)$ is zero-sum. Furthermore, by definition of the $x_{i}$ 's, we have $x_{j} \geq m+1+(j-2)=m+j-1$, so that $B=\left\{x_{1}, \ldots, x_{m}\right\}$ satisfies $g_{j}(B) \geq m+j-2$, and (i) follows.

To prove (ii), suppose $j \geq \frac{m}{p}+p-1$, where $p$ is the smallest prime divisor of $m$. Applying Theorem 2.7 to $P$, it follows that either Theorem 2.7(i) holds and there exist integers $x_{2}, \ldots, x_{m} \in S$ such that $\left(1, x_{2}, x_{3} \ldots, x_{m}\right)$ is zero-sum (note the resulting ( $2 m-$ 2)-set partition from Theorem 2.7(i) will have at most $m-1$ sets with cardinality greater than one; hence since by Theorem 2.7(i) we have that the cardinality of the sumset of that $(2 m-2)$-set partition is at least $m$, then given any one of the $m$ elements from $\mathbb{Z}_{m}$ it follows that we can find a selection of $m-1$ terms from the resulting set partition, including one from each set with cardinality greater than one, which sum to the additive inverse of that element), whence the proof is complete as above; or else Theorem 2.7(ii) holds and there exists a coset, which w.l.o.g. we may assume by translation is a subgroup, say $a \mathbb{Z}_{m}$, such that all but at most $a-2$ terms of the sequence $\Delta S$ are elements of $H_{a}$, whence it follows from Theorem 2.3 that any subset $T \subset S$ satisfying $|T| \geq m+\frac{m}{a}-1+(a-2)$ contains a zero-sum $m$-tuple. Let

$$
S_{1}=\left[m+1, m+\frac{m}{p}+p-2\right] \quad \text { and } \quad S_{2}=[3 m-1,4 m-3] .
$$

Since $\left|S_{1} \cup S_{2}\right|=m+\frac{m}{p}+p-3 \geq m+\frac{m}{a}-1+(a-2)$, it follows that there exist $m$ integers $x_{1}<x_{2}<\cdots<x_{m} \in S_{1} \cup S_{2}$ such that $\sum_{i=1}^{m} \Delta\left(x_{i}\right)=0$. Since $\left|S_{2}\right|=m-1$, we must have $x_{1} \in S_{1}$. Furthermore, since $\left|S_{1}\right|=\frac{m}{p}+p-2 \leq j-1$, we must have $x_{j} \in S_{2}$. Hence it follows that $B=\left\{x_{1}, \ldots, x_{m}\right\}$ is a zero-sum $m$-set satisfying $g_{j}(B) \geq m+j-2$, whence (ii) is satisfied.

Lemma 3.6. Let $m, j$ be positive integers satisfying $2 \leq j \leq m$, let $p$ be the smallest prime divisor of $m$, and let $\Delta:\left[1,6 m+\frac{m}{p}-5\right] \rightarrow \mathbb{Z}_{m}$ be an arbitrary coloring. Then one of the following holds:

(i) there exists a zero-sum $m$-set $B \subset\left[1,6 m+\frac{m}{p}-5\right]$ satisfying $g_{j}(B) \geq m+j-2$;

(ii) there exists an $\left(m, j, \mathbb{Z}_{m}\right)$-solution.

Proof. Let $D$ be the sequence $\left(\Delta\left(m+\frac{m}{p}\right), \Delta\left(m+\frac{m}{p}+1\right), \ldots, \Delta\left(4 m+\frac{m}{p}-4\right)\right)$. In view of the arguments from the third paragraph of the proof of Lemma 3.5, applied to the interval $\left[m+\frac{m}{p}, 4 m+\frac{m}{p}-4\right]$ rather than $[m+1,4 m-3]$, we may assume that there exists a subgroup, say $a \mathbb{Z}_{m}$, such that all but at most $a-2$ terms of $D$ are all elements of $H_{a}$, and, furthermore, that there exists a $(2 m-2)$-set partition $P_{1}$ of the terms of $D$ which are elements of $H_{a}$ such that the sumset of $P_{1}$ is $H_{a}$. Finally, from Theorem 2.1 it follows that from among the sequence

$$
(\Delta(1), \Delta(2), \Delta(3), \cdots, \Delta(a))
$$

we can find a subsequence $D_{1}$ of length $1 \leq q \leq a$ whose terms are consecutive and whose sum is an element $h \in H_{a}$. 
Case 1: $q<j$.

From Proposition 2.5 it follows, by selectively deleting terms from $P_{1}$, that we can find an $(m-q)$-set partition $P_{2}$ of a subsequence $D_{2}$ of $D$ such that the sumset of $P_{2}$ is still $H_{a}$. Consequently, we can find an $m-q$ terms of $D_{2}$ with sum $-h$, which, together with the terms of $D_{1}$, gives an $m$-element zero-sum subset $B$ with $g_{j}(B) \geq m+j-2$.

Case 2: $q \geq j$.

By the arguments in Case 1, we can find an $m$-element zero-sum set $B_{1} \subset\left[1,4 m+\frac{m}{p}-4\right]$ which includes all $q \geq j$ consecutive elements of $D_{1}$, and hence $g_{j}\left(B_{1}\right) \leq j-1$. By Theorem 0 , there exists an $m$-element zero-sum set $B_{2} \subset\left[4 m+\frac{m}{p}-3,6 m+\frac{m}{p}-5\right]$. Since $B_{1}, B_{2}$ are an $\left(m, j, \mathbb{Z}_{m}\right)$-solution, the proof is complete.

Theorem 3.7. Let $m, j$ be integers satisfying $2 \leq j \leq m$.

(i) If $m$ is prime, then $f_{j}\left(m, \mathbb{Z}_{m}\right) \leq 6 m-4$.

(ii) If $j \geq \frac{m}{p}+p-1$, where $p$ is the smallest prime divisor of $m$, then $f_{j}\left(m, \mathbb{Z}_{m}\right) \leq 6 m-4$. (iii) $f_{j}\left(m, \mathbb{Z}_{m}\right) \leq 8 m+\frac{m}{p}-6$.

Proof. Let $s \in\left\{6 m-4,8 m+\frac{m}{p}-6\right\}$, and let $\Delta:[1, s] \rightarrow \mathbb{Z}_{m}$ be an arbitrary coloring. By Theorem 0 , there exists a zero-sum $m$-set $B \subset[1,2 m-1]$, and, furthermore, we have $g_{j}(B) \leq m+j-2$. The proof of (i) and (ii) is complete by letting $s=6 m-4$ and applying Lemma 3.5 (i) or (ii) to $[2 m, s]$, respectively. To show (iii), set $s=8 m+\frac{m}{p}-6$, and apply Lemma 3.6 to $[2 m, s]$.

Corollary 3.8. $\liminf _{m \rightarrow \infty} \frac{F\left(m, \mathbb{Z}_{m}\right)}{F(m, 2)} \leq 1.2$.

Proof. The result follows from Corollary 3.4 and Theorem 3.7(i).

\section{Determination of $f_{m-1}(m, 2)$ and $f_{m-1}\left(m, \mathbb{Z}_{m}\right)$}

For notational convenience, let $f(m, 2)$ and $f\left(m, \mathbb{Z}_{m}\right)$ denote $f_{m-1}(m, 2)$ and $f_{m-1}\left(m, \mathbb{Z}_{m}\right)$, respectively. Furthermore, let $g$ denote the function $g_{m-1}$. Finally, we use the terminology $m$-solution and $\left(m, \mathbb{Z}_{m}\right)$-solution for $(m, m-1)$-solution and $\left(m, m-1, \mathbb{Z}_{m}\right)$-solution, respectively.

Lemma 4.1. Let $m \geq 3$ be an integer, and let $\Delta:[1,3 m-3] \rightarrow\{0,1\}$ be a coloring. Then one of the following holds:

(i) there exists a monochromatic $m$-set $B \subset[1,3 m-3]$ with $g(B) \geq 2 m-4$;

(ii) there exists an m-solution;

(iii) the coloring $\Delta$ is given (up to symmetry) by $1^{m-1} 0^{2 m-3} 1$ or $1^{m-1} 0^{2 m-4} 10$.

Proof. The proof is similar to that of Lemma 3.2 with $j=m-1$, and we omit it.

Lemma 4.2. Let $m \geq 9$ be an integer, and let $\Delta:[1,3 m-3] \rightarrow \mathbb{Z}_{m}$ be a coloring. Then one of the following holds: 
(i) there exists a zero-sum $m$-set $B \subset[1,3 m-3]$ with $g(B) \geq 2 m-3$;

(ii) there exists an $\left(m, \mathbb{Z}_{m}\right)$-solution;

(iii) $\Delta$ is given by $1^{m-2} 21^{m-2} 0^{m}, 1^{m-1} 21^{m-3} 0^{m}$ or $1^{m-3} 21^{m-1} 0^{m}$, up to affine transformation;

(iv) $\Delta$ is given up to affine transformation by $1^{m-1} 0 H$, and there exists $B \subset 0 H$ satisfying $g(B)=2 m-4$;

(v) $\Delta$ reduces to monochromatic.

Proof. Define $S_{1}=\{1,3 m-4,3 m-3\}$ and observe that if there exists a zero-sum $m$-set which uses all the elements of $S_{1}$, then (i) follows. Let $S=[1,3 m-3] \backslash S_{1}$, and let $D$ be the sequence $\Delta(2), \Delta(3), \ldots, \Delta(3 m-5)$. First, we will prove (in Case 1 ) the lemma under a very special coloring, and then we will show that the general problem can be reduced to this special case.

Case 1: $\Delta([1,3 m-3])=\{0,1,2\}$ and $\left|\Delta^{-1}(2)\right|=1$.

Note that $\left|\Delta^{-1}(1)\right| \geq m-2$, as otherwise (v) follows. Therefore there is a zero-sum $m$-set $B$ satisfying $\left|B \cap \Delta^{-1}(2)\right|=1,\left|B \cap \Delta^{-1}(1)\right|=m-2$, and $\left|B \cap \Delta^{-1}(0)\right|=1$ that contains $\{1, a, b\}$, for all distinct $a, b \in[2 m-2,3 m-3]$ such that at most one of the elements of $\{1, a, b\}$ is colored by zero. Hence either $g(B) \geq 2 m-3$ yielding (i), or else every such triple $\{1, a, b\}$ has two of its elements colored by zero. However, this latter case implies either that there exists a monochromatic $m$-set $B$ with $1 \in B$ and $|B \cap[2 m-2,3 m-3]|=m-1$ yielding (i) (if $\Delta(1)=0$ ), or that $\Delta[2 m-2,3 m-3]=0^{m}$, whence $\Delta(1) \in\{1,2\}$. Suppose $\left|\Delta^{-1}(0)\right|=m$. Then it is easy to see that (iii) holds unless there are $m$ consecutive 1's, in which case (ii) follows. Therefore, we may assume that $\left|\Delta^{-1}(0)\right| \geq m+1$. Then $0 \notin \Delta([1, m-1])$ as otherwise (i) follows. Thus $2 \notin \Delta([1, m])$ as otherwise (ii) follows (take for your first set $m-1$ consecutive integers from $[1, m]$ that include an integer colored by 2 along with $\Pi(1,0)$, and for your second set choose any other $m$ integers colored by 0$)$. Hence $\Delta(i)=1$ for $i \in[1, m-1]$ and $\Delta(m)=0$, whence (iv) follows with $B=\{m\} \cup[2 m-1,3 m-3]$.

Case 2: There does not exist $Q \subseteq[1,3 m-3]$ with $|Q|=m+1$ and $|\Delta([1,3 m-3] \backslash Q)|=1$.

Suppose there does not exist $x \in S$ such that $|\Delta(S \backslash x)|=2$. Hence, from the assumption of the case it follows that we can find a $(2 m-5)$-set partition $P$ of the terms of $D$ which has at least $(m-2)$ sets of cardinality 1 , and consequently at most $m-3$ sets with cardinality greater than one. Applying Theorem 2.7 to $P$, we conclude that either Theorem 2.7(i) holds - whence the cardinality of the sumset of the resulting $(2 m-5)$-set partition will be $m$, allowing us to choose a selection of $m-3$ terms (including one from every set with cardinality greater than one) whose sum is the additive inverse of the sum of terms from $S_{1}$, yielding (i) - or else that Theorem 2.7(ii) holds, whence all but at most $a-2+3$ of the elements of $[1,3 m-3]$ are colored by elements from the same coset $\alpha+a \mathbb{Z}_{m}$ of $\mathbb{Z}_{m}$. Hence, Theorem 2.3 implies that any subset of $[1,3 m-3]$ of cardinality $\left(m+\frac{m}{a}-1+a+1\right)$ must contain a zero-sum $m$-set. Thus there is a zero-sum $m$-set

$$
B \subseteq[1, m-2] \cup\left[3 m-4-a-\frac{m}{a}, 3 m-3\right],
$$


and as $\frac{m}{a}+a+2 \leq m-1$ for $m \geq 9$, it follows that

$$
g(B) \geq 3 m-5-a-\frac{m}{a} \geq 2 m-2,
$$

whence (i) follows.

So we may assume that there exists $x \in S$ such that $|\Delta(S \backslash x)|=2$ (i.e., that $S$ is essentially dichromatic). One of the sets $S_{2}=\{2,3 m-5,3 m-6\}, S_{3}=\{3,3 m-5,3 m-6\}$, $S_{4}=\{2,3 m-7,3 m-6\}$ or $S_{5}=\{2,3 m-7,3 m-5\}$, say $S_{3}$, does not contain $x$. Let $\Delta(S)=\left\{\alpha_{1}, \alpha_{2}, \Delta(x)\right\}$ and let $S^{\prime}=[1,3 m-3] \backslash S_{3}$. Apply the arguments of the preceding paragraph to $S^{\prime}$. If some $\alpha_{i}$, say $\alpha_{1}$, colors at most one term in $S^{\prime}$, then $\alpha_{1}$ colors at most $1+\left|S_{3}\right|+\left|S_{1}\right|=7$ integers in total, whence $\alpha_{2}$ colors all but $8 \leq m-1$ integers, yielding (v). Thus we can assume otherwise. Hence, since $x \in S^{\prime}$, it follows that $[1,3 m-3] \backslash\{x\}$ must be colored by the two residue classes $\alpha_{1}$ and $\alpha_{2}$, since otherwise (i) follows. Furthermore, we conclude that $\Delta(x)=\beta \notin\left\{\alpha_{1}, \alpha_{2}\right\}$ as otherwise (v) again follows.

Let $\alpha_{1}-\alpha_{2}=a$. If $(a, m) \neq 1$, then Theorem 2.3 implies that any subset of $[1,3 m-3]$ of cardinality $m+\frac{m}{a}-1+1$ contains a zero-sum $m$-set, whence the proof is complete by the arguments at the end of the first paragraph of Case 2. So, $(a, m)=1$, and hence by an affine transformation we may assume that $\left\{\alpha_{1}, \alpha_{2}\right\}=\{0,1\}$. Furthermore, if $\Delta(x)$ is not equal to 2 or -1 , then there will be a zero-sum $m$-set $B$ satisfying $|B \cap\{x\}|=1$, $\left|B \cap \Delta^{-1}(1)\right|=m-\overline{\Delta(x)} \geq 2$, and $\left|B \cap \Delta^{-1}(0)\right|=\overline{\Delta(x)}-1 \geq 2$ that contains $\{1, a, b\}$ for some distinct $a, b \in[2 m-1,3 m-3]$, and hence $g_{j}(B) \geq 2 m-2$, unless every pair $\{a, b\}$ satisfies $\Delta(1)=\Delta(a)=\Delta(b)$, in which case $B=\{1\} \cup[2 m-1,3 m-3]$ is a monochromatic $m$-set $B$ with $g_{j}(B) \geq 2 m-2$. In both cases (i) follows. Hence, by the affine transformation exchanging 0 and 1 if $\Delta(x)=-1$, this reduces to Case 1 .

Case 3: There exists $Q \subseteq[1,3 m-3]$ such that $|Q|=m+1$ and $|\Delta([1,3 m-3] \backslash Q)|=1$.

Assume w.l.o.g. $\Delta([1,3 m-3] \backslash Q)=\{0\}$. Let $R$ denote a sequence of $m-10$ 's. Define $C=Q \backslash \Delta^{-1}(0)$. Observe that if $|C| \leq m-1$, then (v) follows.

First assume that $|C|=m$. Let $S_{1}$ range over all possible subsequences of $\Delta C$ of length $m-2$. Hence, since $|\Delta(C)| \geq 2$ else (v) follows, then applying Theorem 2.4 to each $S_{1} \cup R$, it follows that there exists a zero-sum subset $C^{\prime} \subset C$ such that $1<\left|C^{\prime}\right| \leq m-2$, unless w.l.o.g. $\Delta(C)=\{1,2\}$ and $\left|\Delta^{-1}(2) \cap C\right|=1$, which reduces to Case 1 . So we may assume such $C^{\prime}$ exists.

Let $y_{1}=\Pi(1,0), y_{2}=\amalg(2,0)$, and $y_{3}=\amalg(1,0)$. Notice that there will be a monochromatic $m$-set $B$ with $g(B) \geq 2 m-3$ unless at least $m-1$ elements of $C$ lie in $\left[1, y_{1}-1\right] \cup\left[y_{2}+1,3 m-3\right]$. Hence, since $2 \leq\left|C^{\prime}\right| \leq m-2$, it follows that $C^{\prime}$ in addition to $m-\left|C^{\prime}\right|$ elements colored by zero, including $y_{1}, y_{2}$ and $y_{3}$ (if $\left|C^{\prime}\right|<m-2$ ) or $y_{1}$ and $y_{3}$ (if $\left|C^{\prime}\right|=m-2, \max \left(C^{\prime}\right)>y_{2}$ ), or $y_{2}$ and $y_{3}\left(\right.$ if $\left|C^{\prime}\right|=m-2, \max \left(C^{\prime}\right)<y_{2}$ ) will form a zero-sum $m$-set $B$ satisfying $g(B) \geq 2 m-3$, yielding (i).

So assume that $|C|=m+1$. As above, we may assume that there exists a zero-sum subset $C^{\prime} \subset C$ such that $2 \leq\left|C^{\prime}\right| \leq m-2$. If $\left|C^{\prime}\right| \geq 3$, then, as in the previous paragraph, it follows that $C^{\prime}$ in addition to $m-\left|C^{\prime}\right|$ elements colored by zero, including $y_{1}, y_{2}$ and $y_{3}$ 
(if $\left|C^{\prime}\right|<m-2$ ) or $y_{1}$ and $y_{3}$ (if $\left|C^{\prime}\right|=m-2, \max \left(C^{\prime}\right)>y_{2}$ ), or $y_{2}$ and $y_{3}$ (if $\left|C^{\prime}\right|=m-2$, $\left.\max \left(C^{\prime}\right)<y_{2}\right)$ will form a zero-sum $m$-set $B$ satisfying $g(B) \geq 2 m-3$, yielding (i). So we can assume all such zero-sum subsets $C^{\prime}$ of $C$ have cardinality two.

Since $m-2 \geq 4$, and since all zero-sums $C^{\prime}$ have cardinality two, it follows that any two such zero-sums must intersect (else the union of two disjoint ones would give a zerosum of size $4 \leq m-2$ ). Suppose the intersection of all the 2-term zero-sum subsets of $C$ is empty. Hence there must be exactly three 2-term zero-sums that pairwise intersect each other with empty three-fold intersection (there can be no more, else there are two disjoint ones, and no fewer, else we contradict the previous sentence). Since this is only possible if all three of these zero-sums are monochromatic in $\frac{m}{2}$, it follows that there are exactly three integers $x_{1}, x_{2}$ and $x_{3}$ colored by $\frac{m}{2}$ (there can be no more, else we have a 4-term zero-sum consisting of four elements colored by $\left.\frac{m}{2}\right)$. Let $Y=C \backslash\left\{x_{1}, x_{2}, y\right\}$, where $y \in C$ is such that $\Delta(y) \neq \frac{m}{2}$. Then $Y$ is colored by at least two distinct residues, including $\frac{m}{2}$. Hence applying Theorem 2.4 to $R \cup Y$ yields a zero-sum $C^{\prime \prime} \subseteq Y \subseteq C$ with $2 \leq\left|C^{\prime \prime}\right| \leq|Y|=m-2$. However, since $x_{1}, x_{2} \notin C^{\prime \prime}$, it follows that $C^{\prime \prime}$ must be distinct from the original three zero-sum subsets, contradicting that $C$ contained exactly three zero-sum subsets of size at most $m-2$. So we may assume there is a term $z \in C$ such that $z$ is contained in every zero-sum subset $C^{\prime} \subseteq C$ with $2=\left|C^{\prime}\right| \leq m-2$.

Applying the arguments of the second paragraph of Case 3 to $C \backslash\{z\}$, we contract the uniqueness of $z \in C^{\prime}$, or we conclude w.l.o.g. that $\Delta(C \backslash\{z\}) \subseteq\{1,2\}$ and $\mid \Delta^{-1}(2) \cap(C \backslash$ $\{z\}) \mid \leq 1$. Since $z$ is one element of a two element zero-sum set, it follows that we must have $\Delta(z)=-1$ or $\Delta(z)=-2$. If $\Delta(z)=-2$, then we can find $C^{\prime}$ with $\Delta C^{\prime}=-21^{2}$, and this reduces to the case $\left|C^{\prime}\right| \geq 3$. So we can assume $\Delta(z)=-1$. Furthermore, we can assume $\left|\Delta^{-1}(2) \cap C\right|=1$, else the affine transformation exchanging 0 and 1 reduces to the hypotheses of Case 1 . Thus $C$ is colored (up to order) by the sequence $1^{m-1}(-1) 2$. Let $z^{\prime}$ be the element colored by 2 .

Hence the pair $\{z, c\}$ is zero-sum for every $c \in C \backslash\left\{z, z^{\prime}\right\}$. Let $z_{1}<z_{2}$ be the first two elements from $C$, and let $z_{3}<z_{4}<z_{5}$ be the last three elements of $C$. As noted before, at least $m-1$ elements of $C$ lie in $\left[1, y_{1}-1\right] \cup\left[y_{2}+1,3 m-3\right]$, so that at most 2 elements of $C$ can lie in $\left[y_{1}, y_{2}\right]$. Since $m-1 \geq 7$, it follows that one of $\left[1, y_{1}-1\right]$ and $\left[y_{2}+1,3 m-3\right]$ must contain at least 4 elements of $C$. Hence, if $\left[1, y_{1}-1\right]$ contains at least 4 elements from $C$, then $y_{2} \geq(3 m-3)-(m+1-4)-2+1=2 m-1$, and we can choose $C^{\prime}$ so that it contains $z_{1}$ or $z_{2}$, whence $C^{\prime}$ in addition to $m-2$ elements colored by zero, including $y_{1}, y_{2}$ and $y_{3}$, will form a zero-sum $m$-set $B$ such that $\min B \leq 2$ and $g(B) \geq 2 m-3$, yielding (i). Therefore we can assume otherwise, whence $\left[y_{2}+1,3 m-3\right]$ must contain at least 4 elements of $C$.

In this case, if $\left|C \cap\left[y_{1}, y_{2}\right]\right|=2$, then we can choose $C^{\prime}$ so that it contains one of $z_{5}$ or $z_{4}$, whence $C^{\prime}$ in addition to $m-2$ elements colored by zero, including $y_{1}, y_{2}$ and $y_{3}$, will form a zero-sum $m$-set $B$ satisfying $g(B) \geq 2 m-3$, yielding (i). Therefore we can assume $\left|C \cap\left[y_{1}, y_{2}\right]\right| \leq 1$. Hence there must be at least $m$ elements of $C$ outside $\left[y_{1}, y_{2}\right]$, at most three less than $y_{1}$ (from the conclusion of the last paragraph), and consequently 
at least $m-4 \geq 5$ elements of $C$ greater than $y_{2}$. Thus $z_{i} \geq y_{2}+3$ for $i \geq 3$, and we must have $z \leq y_{2}+2$, else we can choose $C^{\prime}$ so that it contains $z$ and one of $z_{3}$ or $z_{4}$ or $z_{5}$ that is distinct from $z$, forming, along with $m-2$ integers colored by zero that include $y_{1}, y_{2}$ and $y_{3}$, a zero-sum $m$-set $B$ satisfying $g(B) \geq 2 m-3$, yielding (i). Hence, since there are at least five elements of $C$ greater than $y_{2}$, it follows that at least two of $y_{5}, y_{4}$, and $y_{3}$ must be colored by 1 , say $y_{l_{1}}$ and $y_{l_{2}}$. But then the $m$-set consisting of $y_{l_{1}}, y_{l_{2}}, m-4$ additional elements colored by $1, y_{1}$, and $z^{\prime}$ (recall $\Delta\left(z^{\prime}\right)=2$ ), forms a zero-sum subset $B$ with $g(B) \geq 2 m-3$, completing the proof.

Lemma 4.3. Let $m \geq 5$ be an integer and let $\Delta:[1,5 m-4] \rightarrow \mathbb{Z}_{m}$ be an arbitrary coloring. If there exists an integer $\gamma \geq 2 m$ such that $\Delta([\gamma, \gamma+m-4])=\{z\}$, a zero-sum $m$-set $B_{2} \subset[\gamma, 5 m-4]$ with $g\left(B_{2}\right) \geq 2 m-4$, a zero-sum m-set $B_{3} \subset[\gamma+1,5 m-4]$ with $g\left(B_{3}\right) \geq 2 m-5$, a zero-sum $m$-set $B_{4} \subset\left[\gamma+\left\lfloor\frac{m}{2}\right\rfloor, 5 m-4\right]$ with $g\left(B_{4}\right) \geq m+\left\lceil\frac{m}{2}\right\rceil-4$, an integer $r \geq \gamma+m-3$ such that $\Delta(r)=z$, and a zero-sum $m$-set $B_{5} \subset[r+1,5 m-4]$, then there exists an $\left(m, \mathbb{Z}_{m}\right)$-solution.

Proof. We may w.l.o.g. assume $z=0$. Let $S=[\gamma-2 m+1, \gamma-1], S_{1}=[\gamma-2 m+2, \gamma-1]$ and $S_{2}=[\gamma-2 m+1, \gamma-3] \cup\{\gamma-1\}$. Since $g\left(B_{2}\right) \geq 2 m-4$, we can assume that neither $S_{1}$ nor $S_{2}$ contains a zero-sum $m$-set, whence Theorem $2.4($ a) implies that $|\Delta(S)|=2$. Let $S_{3}=[\gamma-2 m+4, \gamma]$. Since $g\left(B_{3}\right) \geq 2 m-5$, we conclude that there does not exist a zero-sum $m$-set in $S_{3}$, whence, since $|\Delta(S)|=2$, in view of Theorem 2.4 (we remark that the roles/multiplicities of 0,1 and 2 are not as they are in Theorem 2.4) it follows w.l.o.g. that $\Delta(S)=\{1,2\}$ or $\Delta(S)=\{0, b\}$.

Suppose first that $\Delta(S)=\{1,2\}$. Let $\delta$ be the maximal integer such that

$$
s=\sum_{i=\delta}^{\gamma-1} \overline{\Delta(i)} \geq m .
$$

Then $\gamma-m \leq \delta \leq \gamma-\left\lceil\frac{m}{2}\right\rceil$. Notice that $s \in\{m, m+1\}$. Furthermore, if $s=m$, then $B_{1}=\{\delta, \delta+1, \ldots, \delta+m-1\}$ satisfies $g\left(B_{1}\right)=m-2$, whence $B_{1}, B_{4}$ are an $\left(m, \mathbb{Z}_{m}\right)$ solution.

Therefore we may assume that $s=m+1$. Suppose there exists $j \in[\delta, \gamma-1]$ such that $\Delta(j)=1$. If $m$ is even, then $\delta<\gamma-\left\lceil\frac{m}{2}\right\rceil$. On the other hand, if $m$ is odd, then since $s=m+1$, it follows that there are at least two integers colored by 1 in $[\delta, \gamma-1]$, whence $\delta<\gamma-\left\lceil\frac{m}{2}\right\rceil$ as well. Thus $B_{1}=\{\delta, \delta+1, \ldots, \delta+m\} \backslash\{j\}$ is a zero-sum $m$-set satisfying $g\left(B_{1}\right)=m-1$, which together with $B_{4}$ yields an $\left(m, \mathbb{Z}_{m}\right)$-solution.

So we may assume that $\Delta(j)=2$ for $j \in[\delta, \gamma-1]$, whence $m$ is odd as $s=m+1$. Now, we may assume that there exists a maximal integer $\gamma-m \leq \beta \leq \gamma-1$ such that $\Delta(\beta)=1$, since otherwise $B_{1}=\{\gamma-m, \gamma-m+1, \ldots, \gamma-1\}$ is a zero-sum $m$-set satisfying $g\left(B_{1}\right) \leq m-2$, and the proof is complete as in the preceding paragraph. If $\beta \geq \gamma-m+1$, then there exists a zero-sum $m$-set $B \subset\left[\beta, \gamma-1+\frac{m-1}{2}\right]$ satisfying $g(B) \leq \frac{3 m-7}{2}$. But then $B, B_{4}$ are an $\left(m, \mathbb{Z}_{m}\right)$-solution. Therefore, we may assume that $\beta=\gamma-m$, whence 
$\Delta[\gamma-m+1, \gamma-1]=2^{m-1}$. Hence, since $B_{2} \subset[\gamma, 5 m-4]$ is such that $g\left(B_{2}\right) \geq 2 m-4$, it follows that $\Delta(j)=1$ for $j \in[\gamma-2 m+3, \gamma-m]$. But then $B_{1}=[\gamma-2 m+3, \gamma-m+1] \cup\{\gamma\}$ satisfies $g\left(B_{1}\right)=m-2$, whence $B_{1}$ and $B_{4}$ form an $\left(m, \mathbb{Z}_{m}\right)$-solution.

So we may assume that $\Delta(S)=\{0, b\}$. By Theorem 0 , there exists a zero-sum $m$-set $B \subset[\gamma-2 m+1, \gamma-1]$. Since $g\left(B_{2}\right) \geq 2 m-4$, we may assume that $g(B)=2 m-3$, whence $\Delta(\gamma-2 m+1)=\Delta(\gamma-2)=\Delta(\gamma-1)$ and $\left|\Delta^{-1}(\Delta(\gamma-1))\right|=m$. If $\Delta(\gamma-1)=0$, then $B_{1}=\{\gamma-2, \gamma-1, \ldots, \gamma+m-4, r\}$ and $B_{5}$ are an $\left(m, \mathbb{Z}_{m}\right)$-solution. So we may assume that $\Delta(\gamma-1)=b$. Let $y_{1}<y_{2}<\cdots<y_{m-1} \in \Delta^{-1}(0) \cap[\gamma-2 m+2, \gamma-3]$. Then $B_{1}=\left\{y_{1}, y_{2}, \ldots, y_{m-1}, \gamma\right\}$ and $B_{3}$ are an $\left(m, \mathbb{Z}_{m}\right)$-solution.

Theorem 4.4. If $m \geq 9$ is an integer, then $f\left(m, \mathbb{Z}_{m}\right)=f(m, 2)=5 m-4$.

Proof. Since $f(m, 2) \leq f\left(m, \mathbb{Z}_{m}\right)$ holds trivially, in view of Theorem 3.1 it suffices for us to show $f\left(m, \mathbb{Z}_{m}\right) \leq 5 m-4$. Let $\Delta:[1,5 m-4] \rightarrow \mathbb{Z}_{m}$ be an arbitrary coloring. By Theorem 0 , there exists a zero-sum $m$-set $B \subset[1,2 m-1]$ with $g(B) \leq 2 m-3$. Therefore, applying Lemma 4.2 to $S=[2 m, 5 m-4]$, we may assume that neither (i) nor (ii) hold. If (iii) holds, then the proof is complete by Lemma 4.3 with $\gamma=2 \mathrm{~m}$. If (iv) holds, then the proof is again complete by Lemma 4.3 with $\gamma=2 m$ (let $B=B_{i}$ for all $i \in[1,5]$ ). Thus, we may assume that conclusion (v) of Lemma 4.2 holds when applied to $S$. Let $\Delta^{*}: S \rightarrow\{0,1\}$ be the natural induced coloring whose monochromatic $m$-sets are all zero-sum under $\Delta$.

Then we may apply Lemma 4.1 to $S$ and $\Delta^{*}$ and assume that conclusion (ii) does not hold. Suppose first that conclusion (iii) of Lemma 4.1 holds. Then

$$
\Delta^{*} S=0^{m-1} 1^{2 m-4} 01 \quad \text { or } \quad \Delta^{*} S=0^{m-1} 1^{2 m-3} 0,
$$

implying w.l.o.g., since each color class is used at least $m$ times, that

$$
\Delta S=0^{m-1} a^{2 m-4} 0 a \quad \text { or } \quad \Delta S=0^{m-1} a^{2 m-3} 0,
$$

where $a \in \mathbb{Z}_{m}$. From (6) the proof is complete by Theorem 2.4 applied to $[m+2,3 m-2]$ unless $\Delta([m+2,2 m-1])=\{b\}$, where $b \neq 0$, or w.l.o.g. $\Delta([m+2,2 m-1] \backslash\{x\})=\{1\}$, $\Delta(x)=2$, for some $x \in[m+2,2 m-1]$. In the latter case, it can be checked that there is an $m$-element zero-sum subset $B^{\prime} \subset[m+2,3 m-1]$ with $3 m-1 \in B^{\prime}$, and $g\left(B^{\prime}\right) \leq m-1$. Likewise, in the former case if $b \neq a$, then it can be checked that there is an $m$-element zero-sum subset $B^{\prime} \subset[m+2,3 m-1]$ with $g\left(B^{\prime}\right) \leq m-2$. Then, since $2 m-6 \geq m-1$, it follows from $(6)$ that the proof is complete. So $\Delta([m+2,2 m-1])=\{a\}$.

If $[5, m+1] \cap \Delta^{-1}(a) \neq \emptyset$, then there will be an $m$-element monochromatic (in $a$ ) subset $B^{\prime} \subset[5,3 m-1]$, with $g\left(B^{\prime}\right) \leq 2 m-6$, and from (6) the proof will be complete. Hence, in view of Theorem 2.4(b) applied to $[5,2 m+1]$, it follows that $\Delta([5, m+1])=\{0\}$, or else there exists an $m$-element zero-sum subset $B^{\prime} \subseteq[5,2 m+1]$ with $g\left(B^{\prime}\right) \leq 2 m-5$, whence from (6) the proof is complete. So $\Delta([5, m+1])=\{0\}$. Likewise, if $\Delta([1,4]) \nsubseteq\{0, a\}$, then the proof will be complete by applying Theorem 2.4 (b) to both $[1,2 m-4] \cup\{2 m\}$ and $[1,2 m-3]$. So we can conclude $\Delta([1,2 m-1]) \subseteq\{0, a\}$. 
If there exist integers $j_{1}<j_{2} \in[1,4]$ such that $\Delta\left(j_{i}\right)=0$ for $i=1$ and $i=2$, then $B_{1}=\left\{j_{1}, j_{2}, 5,6,7, \ldots, m+1,2 m\right\}$ is a monochromatic $m$-set with $g\left(B_{1}\right) \leq m$, and once more the proof is complete from (6). Therefore, we can assume that there exist integers $j_{1}<j_{2}<j_{3} \in[1,4]$ such that $\Delta\left(j_{i}\right)=a$ for $i=1,2,3$, whence $B_{1}=$ $\left\{j_{1}, j_{2}, j_{3}, m+2, m+3, \ldots, 2 m-2\right\}$ is a monochromatic $m$-set with $g\left(B_{1}\right) \leq 2 m-4$. However, since $\Delta(2 m-1)=a$, it follows from (6) that there exists a monochromatic $m$-set $B_{2} \subset\{2 m-1\} \cup[4 m-3,5 m-4]$ such that $g\left(B_{2}\right) \geq 2 m-4$, and the proof is complete.

So we may assume that conclusion (i) of Lemma 4.1 holds. We consider two cases.

Case 1: There exists $c \in\{0,1\}$ such that $\left|\Delta^{*-1}(c)\right| \leq m-1$.

Without loss of generality $c=1$. It follows that $\left|\Delta^{*-1}(0)\right| \geq 2 m-2$. Furthermore, we may assume that the first $2 m-3$ of the integers colored by 0 are consecutive, since otherwise under $\Delta$ we obtain a zero-sum $m$-set $B_{2}$ satisfying $g\left(B_{2}\right) \geq 2 m-3$, which together with $B$ completes the proof. Applying Lemma 4.3 with $\gamma=\min \left\{\Delta^{-1}(b) \cap S\right\}$, where $b$ is the color such that $\left|\Delta^{-1}(b)\right| \geq 2 m-2$, completes Case 1 .

Case 2: There does not exist $c \in\{0,1\}$ such that $\left|\Delta^{*-1}(c)\right| \leq m-1$.

In this case $|\Delta(S)| \leq 2$ and w.l.o.g. we may assume $\Delta(S)=\{0, a\}$ and that there exist two integers $i_{1}, i_{2} \in[5 m-6,5 m-4]$ such that $\Delta\left(i_{1}\right)=\Delta\left(i_{2}\right)=a$. Hence $x=\min \left\{\Delta^{-1}(a) \cap\right.$ $S\}$ satisfies $x \geq 3 m-2$, as otherwise there will be an $m$-set $B_{2}$ monochromatic in $a$ satisfying $g\left(B_{2}\right) \geq 2 m-3$, which along with $B$ completes the proof. Notice that $x \leq 3 m-1$ as otherwise $[2 m, 3 m-1]$ is a monochromatic $m$-set which along with any $m$ elements colored by $a$ form an $\left(m, \mathbb{Z}_{m}\right)$-solution. But then since conclusion (i) of Lemma 4.1 holds for $[2 m, 5 m-4]$, and since $(5 m-6)-(3 m-1)=2 m-5 \geq m+\left\lceil\frac{m}{2}\right\rceil-4$, it follows, in view of Lemma 4.4 with $\gamma=2 m$ that the proof is complete.

Acknowledgment: The authors would like to thank Professor A. Bialostocki for suggesting that we investigate Conjectures 1.1 and 1.2 and for many fruitful discussions. They would also like to thank the referees for their many helpful suggestions.

\section{References}

[1] N. Alon and M. Dubiner, Zero-sum sets of prescribed size, Combinatorics, Paul Erdös is eighty, Vol. 1, 33-50, Bolyai Soc. Math. Stud., János Bolyai Math. Soc., Budapest, 1993.

[2] A. Bialostocki, Private Communication, June 2002.

[3] A. Bialostocki, Zero sum trees: a survey of results and open problems, Finite and infinite combinatorics in sets and logic (Banff, AB, 1991), 19-29, NATO Adv. Sci. Inst. Ser. C Math. Phys. Scil, 411, Kluwer Acad. Publ., Dordrecht, 1993. 
[4] A. Bialostocki, G. Bialostocki, and D. Schaal, A zero-sum theorem, J. Combin. Theory Ser. A 101 (2003), no. 1, 147-152.

[5] A. Bialostocki, and P. Dierker, On the Erdős-Ginzburg-Ziv theorem and the Ramsey numbers for stars and matchings, Discrete Math. 110 (1992), no. 1-3, 1-8.

[6] A. Bialostocki, and P. Dierker, On zero sum Ramsey numbers: multiple copies of a graph, J. Graph Theory 18 (1994), 143-151.

[7] A. Bialostocki, P. Dierker, D. Grynkiewicz, and M. Lotspeich, On some developments of the Erdős-Ginzburg-Ziv Theorem II, Acta Arith. 110 (2003) no. 2, 173-184.

[8] A. Bialostocki, P. Erdős and H. Lefmann, Monochromatic and zero-sum sets of nondecreasing diameter, Discrete Math. 137 (1995), 19-34.

[9] A. Bialostocki and M. Lotspeich, On Some Developments of the Erdős-Ginzburg-Ziv Theorem I. Sets, graphs and numbers (Budapest, 1991), 97-117, Colloq. Math. Soc. János Bolyai, 60, North-Holland, Amsterdam, 1992.

[10] B. Bollobás and I. Leader, The number of $k$-sums modulo $k$, Journal of Number Theory, 78 (1999), 27-35.

[11] W. Brakemeier, Eine Anzahlformel von zahlen modulo n, Monatsh. Math., 85 (1978), no. 4, 277-282.

[12] Y. Caro, Zero-Sum problems-a survey, Discrete Math. 152 (1996) 93-113.

[13] H. Davenport, On the addition of residue classes, J. London Math. Society 10 (1935), $30-32$.

[14] P. Erdős, A. Ginzburg and A. Ziv, Theorem in additive number theory, Bull. Research Council Israel 10 F (1961) 41-43.

[15] P. Erdős and R. L. Graham, Old and new results in combinatorial number theory, Monographies de L'Enseignement Mathématique, 28, Université de Genève, L'Enseignement Mathématique, Geneva(1980), 128 pp.

[16] C. Flores and O. Ordaz, On the Erdős-Ginzburg-Ziv theorem, Discrete Math., 152 (1996), no. 1-3, 321-324.

[17] Z. Furedi and D. Kleitman, On zero-trees, J. Graph Theory, 16 (1992), 107-120.

[18] L. Gallardo, G. Grekos, L. Habsieger, F. Hennecart, B. Landreau and A. Plagne, Restricted addition in $\mathbb{Z} / n \mathbb{Z}$ and an application to the Erdős-Ginzburg-Ziv problem, J. London Math. Soc., (2) 65 (2002), no. 3, 513-523.

[19] L. Gallardo, G. Grekos and J. Pihko, On a variant of the Erdős-Ginzburg-Ziv problem, Acta Arith., 89 (1999), no. 4, 331-336.

[20] W. D. Gao, Addition theorems for finite abelian groups, J. Number Theory, 53 (1995), no. 2, 241-246.

[21] W. D. Gao and Y. O. Hamidoune, Zero sums in abelian groups, Combin. Probab. Comput., 7 (1998), no. 3, 261-263. 
[22] D. J. Grynkiewicz, On a conjecture of Hamidoune for subsequence sums, Integers, 5 (2005), no. 2, A7, 11 pp. (electronic).

[23] D. J. Grynkiewicz, On a partition analog of the Cauchy-Davenport theorem, Acta Math. Hungar. 107 (2005), no. 1-2, 161-174.

[24] D. J. Grynkiewicz, On four colored sets with nondcecreasing diameter and the ErdösGinzburg-Ziv Theorem, J. Combin. Theory, Ser. A 100 (2002), no. 1, 44-60.

[25] D. J. Grynkiewicz, An extension of the Erdős-Ginzburg-Ziv theorem to hypergraphs, European J. Combin., 26 (2005), no. 8, 1154-1176.

[26] Y. O. Hamidoune, Subsequence Sums, Combinatorics, Propability and Computing, 12 (2003), 413-425.

[27] Y. O. Hamidoune, O. Ordaz and A. Ortuño, On a combinatorial theorem of Erdős, Ginzburg and Ziv, Combin. Probab. Comput., 7 (1998), no. 4, 403-412.

[28] M. Kneser, Ein Satz über abelsche Gruppen mit Anwendungen auf die Geometrie der Zahlen, Math. Z. 64 (1955), 429-434.

[29] H. B. Mann, Two addition theorems, J. Combinatorial Theory, 3 (1967), 233-235.

[30] M. Nathanson, Additive Number Theory, Graduate Texts in Mathematics, 165, Springer-Verlag, New York (1996).

[31] J. E. Olson, An addition theorem for finite abelian groups, Journal of Number Theory, 9 (1977), 63-70.

[32] J. E. Olson, On a combinatorial problem of Erdős, Ginzburg and Ziv, J. Number Theory, 8 (1976), no. 1, 52-57.

[33] A. Schrijver and P. Seymour, A simpler proof and a generalization of the zero-trees theorem, J. Combin. Theory, Ser. A, 58 (1991), 301-305. 